\title{
Effects of Angiotensin Converting Enzyme 2 and Angiotensin Converting Enzyme Genes on Cardiac Remodeling in Rats with Heart Failure
}

\author{
HONG YU WU, YAN QING QI, YONG MAO, CHANG JIANG MAI AND XIAO YUN ZHANG*
}

Department of Cardiac and Vascular Surgery, Ningbo First Hospital, No. 59 Liuting Street, Haishu District, Ningbo, Zhejiang 315012, China

Wu et al.: Angiotensin Converting Enzyme 2 and Angiotensin Converting Enzyme Genes on Cardiac Remodeling

\begin{abstract}
To investigate the effects of angiotensin converting enzyme 2 and angiotensin converting enzyme genes on the cardiac remodeling process in heart failure rats. 38 rats were randomly divided into sham-operated group (S group), heart failure group (H group) and heart failure exercise group (HE group). An incremental load exercise test was used to determine the maximum running speed of the $\mathrm{HE}$ group, while the $\mathrm{S}$ and $\mathrm{H}$ groups maintained their daily activities. The cardiac structure and function and the pathological changes were compared. Compared with $\mathrm{S}$ group, body weight, left ventricular internal diameter at diastole, fractional shortening, left ventricular ejection fraction, myocytes and angiotensin converting enzyme/ angiotensin converting enzyme 2 ratio were decreased while left ventricular weight, left ventricular mass index, ventricular anterior wall diameter at diastole, left ventricular anterior wall diameter at systole, left ventricular posterior wall diameter at diastole, left ventricular posterior wall diameter at systole, atrial natriuretic factor, angiotensin-I, angiotensin-II, messenger RNA and angiotensin converting enzyme, angiotensin converting enzyme 2, transforming growth factor beta protein levels and collagen fibers (i.e. collagen volume fraction) were increased in $\mathbf{H}$ group $(\mathbf{p}<\mathbf{0 . 0 5})$. Compared with $\mathrm{H}$ group, left ventricular weight, left ventricular mass index, left ventricular internal diameter at diastole, fractional shortening, left ventricular ejection fraction, myocytes were increased while collagen volume fraction, atrial natriuretic factor, angiotensin-I, angiotensin-II, messenger RNA and angiotensin converting enzyme, angiotensin converting enzyme 2, transforming growth factor beta protein levels were reduced and angiotensin converting enzyme/angiotensin converting enzyme 2 ratio were decreased $(\mathbf{p}<\mathbf{0 . 0 5})$. Long-term aerobic exercise attenuated myocardial fibrosis and improved cardiac function by inhibiting collagen synthesis and promoting its degradation and cardiac remodeling was inhibited and improved by a mechanism that may be related to partial restoration of angiotensin converting enzyme 2/angiotensin converting enzyme signalling function.
\end{abstract}

Key words: Heart failure, cardiac remodeling, angiotensin converting enzyme, angiotensin converting enzyme 2

Cardiac remodeling, is an important cause of the occurrence and development of chronic heart failure (CHF), the main pathophysiological mechanisms include embryonic gene activation, myocardial hypertrophy and apoptosis ${ }^{[1]}$. Overexpression of reninangiotensin system (RAS) is one of the mechanisms of myocardial fibrosis induced by heart failure and plays an important role in cardiac remodeling. As the core enzyme of RAS, angiotensin converting enzyme (ACE) catalyzes the production of angiotensin-I (Ang-I) and angiotensin-II (Ang-II), which are the key effectors of RAS. In 2000, Donoghue et al. ${ }^{[2]}$ first discovered the homologue of ACE, named angiotensin converting enzyme 2 (ACE2). The main biological effect of ACE2 is to transform Ang-II into Ang 1-7. Therefore, ACE and ACE2 is a pair of enzymes with opposite functional

*Address for correspondence

E-mail: xx8709nihai@163.com 
effects. Exercise training, especially low-intensity aerobic exercise, is an important means of rehabilitation for patients with heart failure, which can improve heart function and improve the quality of life of patients ${ }^{[3]}$, which may be related to inhibiting heart remodeling ${ }^{[4]}$, but the specific mechanism is not clear ${ }^{[5,6]}$. The aim of this study was to investigate the effects of $10 \mathrm{w}$ aerobic exercise on cardiac structure, cardiac function, collagen content and ACE2 and ACE2 gene expression in Wistar rats with heart failure induced by coronary artery ligation and to explore the effects of exercise on cardiac remodeling and $\mathrm{ACE}$ and $\mathrm{ACE} 2$ gene expression and to further study the role of ACE and ACE2 in heart failure.

\section{MATERIALS AND METHODS}

\section{Establishment of heart failure model:}

A total of 38 healthy male specific-pathogen free (SPF) Wistar rats aged 2 mo were selected, weighing 250-280 g (purchased from Guangdong Chunsheng Biotechnology Development Co., Ltd.). Twenty eight rats were randomly selected to establish heart failure models: $1 \%$ Pentobarbital Sodium anesthesia, supine fixation, thoracotomy on the left side of the sternum between the third and fourth ribs to expose the heart, ligate the left anterior descending coronary artery with 0 silk thread 2-3 mm below the left atrial appendage, quickly put back the heart, close the chest and suture the chest wall. The other 10 rats were treated with sham operation, that is, only suture was ligated after thoracotomy and other operations were the same as above. $4 \mathrm{w}$ later, echocardiography was performed and left ventricular ejection fraction (LVEF) $\leq 45 \%$ was used as the mark of successful modeling ( 2 animals failed to model, 1 died after modeling). The animals were divided into sham operation control group (S group, $\mathrm{n}=10$ ), heart failure control group ( $\mathrm{H}$ group, $\mathrm{n}=12$ ) and heart failure exercise group (HE group, $n=13$ ). Group $\mathrm{S}$ and group $\mathrm{H}$ kept quiet, while $\mathrm{HE}$ group rats received treadmill training for $10 \mathrm{w}$.

\section{Exercise program:}

First, the maximum running speed of rats in HE group was determined by incremental load exercise test ${ }^{[6]}$. The program was: warm up for $15 \mathrm{~min}$ (slope $0^{\circ}$ speed $5 \mathrm{~m} /$ min) and rest for 5 min to carry out incremental load test. The initial load was $7 \mathrm{~m} / \mathrm{min}$ and the maximum running speed was increased by $5 \mathrm{~m} / \mathrm{min}$ every $3 \mathrm{~min}$ (slope $0^{\circ}$ until exhaustion). The rats in HE group were trained on treadmill for $10 \mathrm{w}$. The running speed was 50 $\%$ of the maximum running speed in the first $2 \mathrm{w}$ and 60 $\%$ of the maximum running speed in the last $8 \mathrm{w}$. The time was $30 \mathrm{~min}$ on the first day and then increased by $10 \mathrm{~min}$ every day until $60 \mathrm{~min} / \mathrm{d}$. The frequency was 5 $\mathrm{d} / \mathrm{w}$.

\section{Detection of cardiac structure and function:}

After abdominal anesthesia, the animals were fixed on their back. The structure and function of the heart were measured by small animal ultrasonic instrument (visualSonics vevo770, Canada). The parameters included left ventricular internal diameter at diastole (LVIDd), left ventricular internal diameter at systole (LVIDs), left ventricular anterior wall diameter at systole (LVAWDs), left ventricular posterior wall diameter at diastole (LVPWDd), left ventricular posterior wall diameter at systole (LVPWDs), left ventricular fractional shortening (LVFS) and LVEF.

\section{Heart tissues collection:}

The left ventricular weight (LVW) and right ventricular weight (RVW) were measured respectively and the left ventricular mass index (LVMI) (LVMI=LVW/ body weight (BW) was calculated. The left ventricle was divided into two parts. One part was measured for collagen content and the other part was quickly placed in liquid nitrogen and transferred at $-80^{\circ}$ refrigerator for gene expression.

\section{Determination of collagen content in heart tissue:}

The left ventricular myocardium was fixed in $10 \%$ formalin, dehydrated, transparent, embedded and sectioned $(5 \mu \mathrm{m})$ before Masson staining. Five visual fields were randomly selected from each slice and myocardial collagen area was calculated by image analysis software. The percentage of collagen tissue area in the measured visual field area was called collagen volume fraction (CVF).

\section{Detection of gene expression level:}

The total ribonucleic acid (RNA) was extracted by TRIzol method and the complementary deoxyribonucleic acid (cDNA) was obtained by reverse transcription. The messenger RNA (mRNA) expression of Ang I, Ang II and atrial natriuretic factor (ANF) was measured by real-time fluorescence quantitative polymerase chain reaction (PCR) (ABI 7900 fluorescence quantitative PCR instrument, USA). The amplification conditions were: pre denaturation $95^{\circ} / 1 \mathrm{~min} ; 95^{\circ} / 15 \mathrm{~s}, 55^{\circ} / 15$ $\mathrm{s}, 72^{\circ} / 15 \mathrm{~s}$, a total of 40 cycles. Using $\beta$-actin as internal reference, the relative expression of target gene (multiple of S group) was calculated. The primer sequence was shown in Table 1. 
TABLE 1: GENE PRIMER SEQUENCES

\begin{tabular}{|c|c|c|}
\hline Genes & Primer (bp) & Product size \\
\hline \multirow{2}{*}{ Ang-I } & F: 5'-TTGACCCTAACCAAGGATGC-3' & \multirow{2}{*}{197} \\
\hline & R: 5'-TGGGCTCCAATCCTGTCAATC-3' & \\
\hline \multirow{2}{*}{ Ang-II } & F: 5'-AGATGGACTAAGTGGACATC-3' & \multirow{2}{*}{450} \\
\hline & R: 5'-CATGTTTCTCCGGTTTCCAT-3' & \\
\hline \multirow{2}{*}{ ANF } & F: 5' -CATCACCCTGGGCTTCTTCCT-3' & \multirow{2}{*}{406} \\
\hline & R: 5'-AGATGGACTAAGTGGACATC-3' & \\
\hline \multirow{2}{*}{ B-actin } & F: 5'-AGACCTTCAACACCCCAG-3' & \multirow{2}{*}{316} \\
\hline & R: 5' -CACGATTTCCCTCTCAGC-3' & \\
\hline
\end{tabular}

\section{RESULTS AND DISCUSSION}

Comparison of cardiac structure and function of groups was measured. Compared with group S, BW, LVIDd, fractional shortening (FS) and LVEF in group $\mathrm{H}$ decreased $(p<0.05)$, while LVW, LVMI, left ventricular anterior wall diameter at diastole (LVAWDd), LVAWDs, LVPWDd and LVPWDs increased $(\mathrm{p}<0.05)$. Compared with group H, LVW, LVMI, LVIDd, FS and LVEF increased in group HE $(\mathrm{p}<0.05)$, as shown in Table 2 .

TABLE 2: COMPARISON OF CARDIAC STRUCTURE AND FUNCTION

\begin{tabular}{lccc}
\hline & $\mathrm{S}(\mathrm{n}=10)$ & $\mathrm{H}(\mathrm{n}=12)$ & $\mathrm{HE}(\mathrm{n}=13)$ \\
\hline BW $(\mathrm{g})$ & $430 \pm 38$ & $316 \pm 36^{*}$ & $337 \pm 45$ \\
LVW (me) & $802 \pm 105$ & $988 \pm 112^{*}$ & $1178 \pm 118^{\#}$ \\
RVW (mg) & $185 \pm 19$ & $178 \pm 20$ & $185 \pm 24$ \\
LVMK (mg/g) & $1.89 \pm 0.26$ & $3.16 \pm 0.50^{*}$ & $3.59 \pm 0.52^{\#}$ \\
LVHM (mm) & $8.5 \pm 1.3$ & $7.2 \pm 1.0^{*}$ & $8.6 \pm 1.2^{\#}$ \\
LVIDs (mm) & $5.3 \pm 0.8$ & $5.1 \pm 1.1$ & $5.4 \pm 0.9$ \\
LVAWDd (mm) & $1.3 \pm 0.3$ & $1.9 \pm 0.2^{*}$ & $1.8 \pm 0.2$ \\
LVAWDs (mm) & $2.6 \pm 0.4$ & $3.2 \pm 0.7^{*}$ & $3.0 \pm 0.9$ \\
LVPWDd (mm) & $1.4 \pm 0.2$ & $2.2 \pm 0.5^{*}$ & $2.2 \pm 0.6$ \\
LVPWDs (mm) & $2.2 \pm 0.5$ & $2.8 \pm 0.8^{*}$ & $2.6 \pm 0.7$ \\
FS (mm/s) & $47.6 \pm 6.7$ & $31.2 \pm 4.6^{*}$ & $38.8 \pm 3.9^{\#}$ \\
LVEF $(\%)$ & $56.5 \pm 7.6$ & $38.5 \pm 5.7^{*}$ & $47.2 \pm 5.8^{\#}$ \\
\hline Note:Compared
\end{tabular}

Note: Compared with sham operation control group, ${ }^{*} \mathrm{p}<0.05$; compared with heart failure control group, ${ }^{*} p<0.05$

Comparison of histopathological changes of heart was done. In $\mathrm{S}$ group hearts, the myocardial fibers were evenly stained without collagen component; while in $\mathrm{H}$ group, the myocardial cells were decreased, the collagen component was significantly increased and the degree of fibrosis was obvious and the collagen fiber $(\mathrm{CVF})$ was higher than that in group $\mathrm{S}(\mathrm{p}<0.05)$; compared with group $\mathrm{H}$, the myocardial cells in group $\mathrm{HE}$ were increased and arranged orderly and the CVF was significantly decreased $(\mathrm{p}<0.05)$, as shown in Table 3.

TABLE 3: COMPARISON OF HISTOPATHOLOGICAL CHANGES OF HEART

\begin{tabular}{cccc}
\hline & $S(n=10)$ & $H(n=12)$ & $H E(n=13)$ \\
\hline Collagen fiber & $6.5 \% \pm 1.1 \%$ & $24.3 \% \pm 5.8 \% *$ & $15.7 \% \pm 3.9 \%{ }^{*}$ \\
\hline
\end{tabular}

Note: Compared with sham operation control group, ${ }^{*} \mathrm{p}<0.05$; compared with heart failure control group, ${ }^{*} \mathrm{p}<0.05$

Comparison of gene expression changes was observed. Compared with group $\mathrm{S}$, the mRNA levels of ANF, Ang-I and Ang-II, the protein levels of ACE, ACE2 and transforming growth factor beta (TGF- $\beta$ ) in group $\mathrm{H}$ were increased $(p<0.05)$ and the ratio of ACE/ACE2 was decreased $(p<0.05)$. Compared with group $H$, the mRNA levels of ANF, Ang-I and Ang-II, the protein levels of ACE, ACE2 and TGF- $\beta$ in group HE were decreased $(p<0.05)$ and the ratio of ACE/ACE2 was increased $(p<0.05)$, as shown in Table 4 and fig. 1 . The protein expression was determined by western blot.

TABLE 4: COMPARISON OF GENE EXPRESSION CHANGES

\begin{tabular}{llll}
\hline & $S(n=10)$ & $H(n=12)$ & $H E(n=13)$ \\
\hline mRNA & & & \\
Ang-I & $1.00 \pm 0.10$ & $3.78 \pm 0.65^{*}$ & $1.59 \pm 0.20^{\#}$ \\
Ang-II & $1.02 \pm 0.12$ & $2.67 \pm 0.56^{*}$ & $1.86 \pm 0.55^{\#}$ \\
ANF & $0.99 \pm 0.15$ & $3.39 \pm 0.68^{*}$ & $1.80 \pm 0.36^{\#}$ \\
Proteins & & & \\
ACE & $0.99 \pm 0.12$ & $1.76 \pm 0.16^{*}$ & $1.20 \pm 0.10^{\#}$ \\
ACE2 & $1.01 \pm 0.11$ & $3.19 \pm 0.40^{*}$ & $1.55 \pm 0.16^{\#}$ \\
TGF-B & $1.00 \pm 0.10$ & $2.80 \pm 0.30^{*}$ & $1.70 \pm 0.20^{\#}$ \\
ACE/ACE2 & $1.00 \pm 0.11$ & $0.52 \pm 0.08^{*}$ & $0.80 \pm 0.09^{\#}$ \\
\hline
\end{tabular}

Note: Compared with sham operation control group, ${ }^{*} \mathrm{p}<0.05$; compared with heart failure control group, ${ }^{*} \mathrm{p}<0.05$
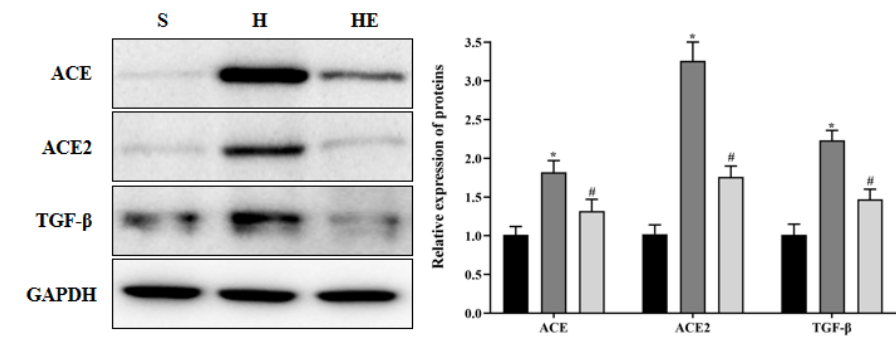

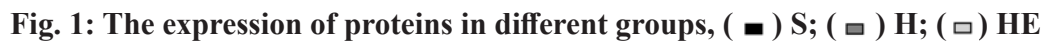


In this study, we successfully established a heart failure model after myocardial infarction, which showed that the heart weight (LVW, LVMI) increased, the heart cavity decreased (LVIDd decreased), the heart wall thickened (LVAWDd, LVAWDs, LVPWDd and LVPWDs increased). At the gene level, the embryonic gene (ANF) is reactivated; the heart is remolded and eventually leads to the progressive decline of heart function (FS and LVEF). After $10 \mathrm{w}$ of aerobic exercise, compared with group $\mathrm{H}$, the LVW, LVMI and inner diameter of cardiac cavity (LVIDd) of group HE increased and the thickness of ventricular wall did not change significantly, that is, the "centrifugal hypertrophy" of left ventricle occurred, which may be caused by the increase of cardiac blood volume (preload or volume load) after aerobic exercise ${ }^{[7,8]}$. The down-regulation of ANF expression indicates that the abnormal activation of embryonic genes is significantly inhibited and the increase of FS and LVEF indicates the increase of myocardial contractility. These results suggest that exercise-induced left ventricular physiological remodeling and pathological remodeling exist simultaneously and compete with each other in the process of exercise rehabilitation in rats with heart failure ${ }^{[9]}$. Finally, the benign effect of the former reverses the negative effect of the latter, showing that the left ventricular structure changes from pathological hypertrophy to physiological hypertrophy and the cardiac function is improved. Therefore, long-term aerobic exercise can improve cardiac function by inhibiting cardiac remodeling in heart failure. In heart failure, excessive deposition of myocardial extracellular matrix (ECM) and decreased compliance of ventricular wall can easily lead to fatal arrhythmia, indicating that myocardial fibrosis plays an important role in cardiac remodeling ${ }^{[10]}$. Myocardial ECM is mainly controlled by Ang-I and Ang-II and its physiological function is to provide support, conduct stress and ensure myocardial compliance ${ }^{[11]}$. In this study, the mRNA expressions of Ang-I and Ang-II were up-regulated in group H. Masson staining showed a significant increase in collagen composition and CVF, suggesting excessive collagen deposition and myocardial fibrosis in heart failure. After aerobic exercise, the mRNA expression of Ang-I and Ang-II in HE group was significantly lower than that in $\mathrm{H}$ group and CVF was significantly reduced, which was consistent with the research results of Ferrario et al. ${ }^{[12]}$, indicating that aerobic exercise can reduce myocardial collagen deposition and inhibit cardiac remodeling. In addition, Grobe et al. ${ }^{[13]}$ found that $12 \mathrm{w}$ treadmill training can improve the cardiac function of aging rats and reduce the cross-linking of myocardial collagen, suggesting that exercise has a benign effect on collagen content, spatial arrangement and interaction of collagen.

Collagen metabolism is mainly regulated by ACE, ACE2 and TGF- $\beta$. Matrix metalloproteinase (MMP) is a proteolytic enzyme of collagen degradation. Many ACEs can be expressed in myocardium, among which ACE is mainly responsible for the degradation of Ang-I and Ang-II. ACE2 is an endogenous inhibitor of ACE, which is inactivated by binding with ACE. ACE and ACE2 are in a state of dynamic balance, regulating the synthesis, metabolism and renewal of collagen. TGF- $\beta$ is closely related to cardiovascular disease. The specific expression of TGF- $\beta$ in myocardium can lead to myocardial hypertrophy and fibrosis ${ }^{[14]}$. Endothelin, Ang-II and other factors that induce myocardial fibrosis can lead to the increase of TGF- $\beta$, which indicates that the up regulation of TGF- $\beta$ is the common pathway of myocardial fibrosis induced by many factors. Recent studies have found that TGF- $\beta$ is a signal molecule upstream of ACE $2^{[15]}$. TGF- $\beta$ participates in the process of cardiac remodeling by up regulating the expression of ACE2, while reducing the expression of TGF- $\beta$ can reduce the accumulation of myocardial collagen ${ }^{[16]}$. The expression of TGF- $\beta$ and ACE2 is up-regulated in heart failure $^{[17]}$, but the change of ACE expression in cardiac remodeling is still controversial. Some scholars believe that the down-regulation of ACE expression leads to collagen accumulation and myocardial fibrosis ${ }^{[18]}$. However, Mukherjee et al. ${ }^{[19]}$ found that myocardial ACE was significantly increased in heart failure. Oyamada et al. ${ }^{[20]}$ confirmed that the expression of ACE was up-regulated in ischemia-reperfusion injury. Excessive decomposition of collagen by ACE led to the degradation of myocardial interstitium, which led to the disorder of myocardial arrangement, myocardial dilation and development of ventricular cavity enlargement and eventually cardiac remodeling. The difference of the above conclusions may be related to the experimental model, the degree of heart failure and the duration of heart failure. In this study, compared with group $H$, the expressions of TGF- $\beta$, ACE2 and $\mathrm{ACE}$ protein in $\mathrm{HE}$ group were significantly upregulated, while the ratio of ACE/ACE2 was decreased, suggesting that the inhibition of ACE2 was enhanced, the relative activity of ACE was decreased and the ability of collagen degradation was decreased. The up regulation of $\mathrm{ACE}$ expression may be a compensatory reaction and the balance of collagen metabolism is ultimately determined by ACE/ACE2 ratio $^{[21]}$. After $10 \mathrm{w}$ of aerobic exercise, the expressions of TGF- $\beta$, 
ACE2 and ACE in HE group were decreased, while the ratio of $\mathrm{ACE} / \mathrm{ACE} 2$ was increased, suggesting that the inhibitory effect of ACE2 on ACE was partially relieved. These results suggest that long-term aerobic exercise can regulate the dynamic balance of ACE/ ACE2, reduce collagen deposition and myocardial fibrosis by inhibiting the overexpression of TGF- $\beta$, thus inhibiting cardiac remodeling in heart failure. In conclusion, ACE2/ACE signaling pathway is involved in the process of exercise improving myocardial remodeling, which may be one of the mechanisms of exercise protecting heart from heart failure.

Cardiac hypertrophy, decreased cardiac function and myocardial remodeling after heart failure are related to collagen deposition caused by abnormal ACE2/ ACE signal transduction. Long term aerobic exercise can reduce myocardial fibrosis and improve cardiac function by inhibiting collagen synthesis and promoting its degradation. Cardiac remodeling can be inhibited and improved. The mechanism may be related to the partial recovery of ACE2/ACE signal function.

\section{Acknowledgements:}

This work was supported by the Ningbo First Hospital.

\section{Conflicts of interest:}

The authors declared no conflict of interest.

\section{REFERENCES}

1. Johnson FL. Pathophysiology and etiology of heart failure. Cardiol Clin 2014;32(1):9-19.

2. Donoghue M, Hsieh F, Baronas E, Godbout K, Gosselin M, Stagliano N, et al. A novel angiotensin-converting enzymerelated carboxypeptidase (ACE2) converts angiotensin I to angiotensin 1-9. Circ Res 2000;87(5):e1-9.

3. Crackower MA, Sarao R, Oudit GY, Yagil C, Kozieradzki I, Scanga SE, et al. Angiotensin-converting enzyme 2 is an essential regulator of heart function. Nature 2002;417:822-8.

4. Diez-Freire C, Vazquez J, Correa de Adjounian MF, Ferrari MF, Yuan L, Silver X, et al. ACE2 gene transfer attenuates hypertension-linked pathophysiological changes in the SHR. Physiol Genomics 2006;27(1):12-9.

5. Ehlers MR, Riordan JF. Angiotensin-converting enzyme: zincand inhibitor-binding stoichiometries of the somatic and testis isozymes. Biochemistry 1991;30(29):7118-26.

6. Amenta F, Di Tullio MA, Tomassoni D. Arterial hypertension and brain damage - evidence from animal models. Clin Exp Hypertens 2003;25(6):359-80.

7. Benter IF, Diz DI, Ferrario CM. Pressor and reflex sensitivity is altered in spontaneously hypertensive rats treated with angiotensin-(1-7). Hypertension 1995;26(6):1138-44.

8. Benter IF, Yousif MH, Anim JT, Cojocel C, Diz DI. Angiotensin-(1-7) prevents development of severe hypertension and end-organ damage in spontaneously hypertensive rats treated with L-NAME. Am J Physiol Heart Circ Physiol 2006;290(2):H684-91.
9. Burrell LM, Risvanis J, Kubota E, Dean RG, MacDonald PS, $\mathrm{Lu} \mathrm{S}$, et al. Myocardial infarction increases ACE2 expression in rat and humans. Eur Heart J 2005;26(4):369-75.

10. Danilczyk U, Eriksson U, Oudit GY, Penninger JM. Physiological roles of angiotensin-converting enzyme 2. Cell Mol Life Sci 2004;61(21):2714-9.

11. Der Sarkissian S, Huentelman MJ, Stewart J, Katovich MJ, Raizada MK. ACE2: A novel therapeutic target for cardiovascular diseases. Prog Biophys Mol Biol 2006;91(12):163-98.

12. Ferrario CM, Trask AJ, Jessup JA. Advances in biochemical and functional roles of angiotensin-converting enzyme 2 and angiotensin-(1-7) in regulation of cardiovascular function. Am J Physiol Heart Circ Physiol 2005;289(6):H2281-90.

13. Grobe JL, Mecca AP, Mao H, Katovich MJ. Chronic angiotensin-(1-7) prevents cardiac fibrosis in DOCA-salt model of hypertension. Am J Physiol Heart Circ Physiol 2006;290(6):H2417-23.

14. Hao X, Zhang Y, Zhang X, Nirmalan M, Davies L, Konstantinou $\mathrm{D}$, et al. TGF- $\beta 1$-mediated fibrosis and ion channel remodeling are key mechanisms in producing the sinus node dysfunction associated with SCN5A deficiency and aging. Circ Arrhythm Electrophysiol 2011;4(3):397-406.

15. Ji H, Tang H, Lin H, Mao J, Gao L, Liu J, et al. Rho/Rock cross-talks with transforming growth factor- $\beta /$ Smad pathway participates in lung fibroblast-myofibroblast differentiation. Biomed Rep 2014;2(6):787-92.

16. Chen R, Xue J, Xie ML. Reduction of isoprenaline-induced myocardial TGF- $\beta 1$ expression and fibrosis in osthole-treated mice. Toxicol Appl Pharmacol 2011;256(2):168-73.

17. Kapur NK. Transforming growth factor- $\beta$ : governing the transition from inflammation to fibrosis in heart failure with preserved left ventricular function. Circ Heart Fail 2011;4(1):57.

18. Deatrick KB, Luke CE, Elfline MA, Sood V, Baldwin J, Upchurch Jr GR, et al. The effect of matrix metalloproteinase 2 and matrix metalloproteinase 2/9 deletion in experimental post-thrombotic vein wall remodeling. J Vasc Surg 2013;58(5):1375-84.

19. Mukherjee R, Snipes JM, Saunders SM, Zavadzkas JA, Spinale FG. Discordant activation of gene promoters for matrix metalloproteinases and tissue inhibitors of the metalloproteinases following myocardial infarction. J Surg Res 2012;172(1):59-67.

20. Oyamada S, Bianchi C, Takai S, Chu LM, Sellke FW. Chymase inhibition reduces infarction and matrix metalloproteinase-9 activation and attenuates inflammation and fibrosis after acute myocardial ischemia/reperfusion. J Pharmacol Exp Ther 2011;339(1):143-51.

21. Komatsu T, Suzuki Y, Imai J, Sugano S, Hida M, Tanigami A, et al. Molecular cloning, mRNA expression and chromosomal localization of mouse angiotensin-converting enzyme-related carboxypeptidase (mACE2). DNA Seq 2002;13(4):217-20.

This is an open access article distributed under the terms of the Creative Commons Attribution-NonCommercial-ShareAlike 3.0 License, which allows others to remix, tweak, and build upon the work non-commercially, as long as the author is credited and the new creations are licensed under the identical terms

This article was originally published in a special issue,

"Therapeutic Perspectives in Biomedical Research and Pharmaceutical Sciences and their Nursing Methods"

Indian J Pharm Sci 2021:83(4)Spl issue "122-126" 